\title{
Development and Testing of High Surface Area Iridium Anodes for Molten Oxide Electrolysis.
}

\author{
Anatoliy Shchetkovskiy and Timothy McKechnie \\ Plasma Processes Inc., Huntsville, AL, 35811 \\ Donald R. Sadoway and James Paramore \\ Massachusetts Institute of Technology, Cambridge, MA, 02139 \\ Orlando Melendez \\ NASA KSC, Kennedy Space Center, FL, 32899 \\ Peter A. Curreri \\ NASA MSFC, Marshall Space Flight Center, AL, 35899
}

\begin{abstract}
Processing of lunar regolith into oxygen for habitat and propulsion is needed to support future space missions. Direct electrochemical reduction of molten regolith is an attractive method of processing, because no additional chemical reagents are needed. The electrochemical processing of molten oxides requires high surface area, inert anodes. Such electrodes need to be structurally robust at elevated temperatures $\left(1400-1600^{\circ} \mathrm{C}\right)$, be resistant to thermal shock, have good electrical conductivity, be resistant to attack by molten oxide (silicate), be electrochemically stable and support high current density. Iridium with its high melting point, good oxidation resistance, superior high temperature strength and ductility is the most promising candidate for anodes in high temperature electrochemical processes. Several innovative concepts for manufacturing such anodes by electrodeposition of iridium from molten salt electrolyte (EL-Form ${ }^{\mathrm{TM}}$ process) were evaluated. Iridium electrodeposition to form of complex shape components and coating was investigated. Iridium coated graphite, porous iridium structure and solid iridium anodes were fabricated. Testing of electroformed iridium anodes shows no visible degradation. The result of development, manufacturing and testing of high surface, inert iridium anodes will be presented.
\end{abstract}

